\title{
Strategic Framework to Promote Rationale Use of Medicine in Lmics: A Policy Perspective \\ Sathyanarayana Tamysetty*
}

Indian Institute of Public Health-Bangalore Campus, India

*Corresponding author: Dr. Sathyanarayana TN, Indian Institute of Public Health-Bangalore Campus, India, Tel: +91-9535450999

\begin{abstract}
Introduction: Through the application of a strategic framework, the paper attempts to sharpen the focus, coordination and dissemination of promotion of rational use of medicines (PRUM) framework.

Purpose of the framework: The purpose of this strategic framework is to guide and organize the systematic planning, implementation, and evaluation of irrational use of medicines and other efforts aimed at improving rational use of medicines and reducing and, ultimately, eliminating irrational use of medicines.

Aim: The suggested framework efforts include those aimed directly at irrational use of medicines and related health problems, but also those that support a "systems approach" to addressing such problems across the country. These systems approaches have not been previously available in efforts targeted towards PRUM and improve health issues.

Methodology: The logic framework model approach being adopted to enhance the clarity, for developing the strategic framework.

Conclusions: The Framework may provide a rational basis for identifying and developing effective strategies, practices, and other efforts that could conduct and supports promotion of rational use of medicines with range of stakeholders across the country. The Framework does this by drawing on existing science and knowledge about the nature of deterring factors and suggests sustainable interventions to promote 'promotion of rational use of medicines'.
\end{abstract}

\section{Keywords}

Rational use of medicines, Policy to promote rational use of medicines, Framework

\section{Introduction}

Worldwide, the irrational use of medicines is a major problem. As per WHO estimates, more than half of all medicines are prescribed, dispensed or sold inappropriately, and that half of all patients fail to take them correctly [1]. Across low and middle income countries (LMICs), this issue has been impeding health goal achievement while attempts are being made to overcome the same [2]. While health gains has been made for more than six decades since the 1947 when India got independence, there is persistence of several health problems and issues related to health services promotion such as irrational usage of medicines. This overuse, underuse or misuse of medicines is practiced in India and LMICs by patients, practitioners and pharmaceutical companies which in turn results in wastage of scarce resources and widespread health hazards [3-6]. The current approach and strategies are not producing the kind of results needed for ensuring the promotion of rational use of medicines (PRUM). It is imperative that LMICs adopts appropriate health policies and programs across the spectrum of measures needed in order to eliminate the irrational use of medicines. For achieving the optimal rational usage of medicines in consistent fashion, we hereby propose a multipronged strategic frame work encompassing the complete range of stakeholders to achieve the same.

\section{Need of a framework}

Given the nature of extensive practices of irrational usage of medicine in India and LMIC, there is no single strategic approach, which can connect and address the diverse nature of PRUM. In order to achieve the objective of rational usage of medicines and to reduce the complexity related to irrational usage of medicines across the different stakeholders 
such as individuals, community, practitioners, health administrators and policy makers, there is need of clear inter-connected logical approach [2,7-9]. In this context, we propose this "Strategic Framework for Improving Rational Use of Medicines" in LMICs. The proposed framework is timely and relevant and it is intended to help, guide, organize and coordinate systematic planning, implementation and evaluation of efforts within broad health system [10]. Further, the proposed framework reflects understanding of current knowledge, nature of irrational use of medicines, causes or contributing factors and thereby, evolves solutions and desired outcomes in sustainable fashion [11].

\section{Methodology}

The four-step logic framework model approach is adopted in this paper [10]. First step involved studying long term and short term factors contributing to existing problems.

Second step involved in evaluating existing strategies and practices and supporting resources. Third step involved in developing measurable outcomes and impact and final step involved to enumerate long-term objectives and goals. Each step involved extensive literature review and targeted selective reviews of the literature from fields including public health and medicine [12].

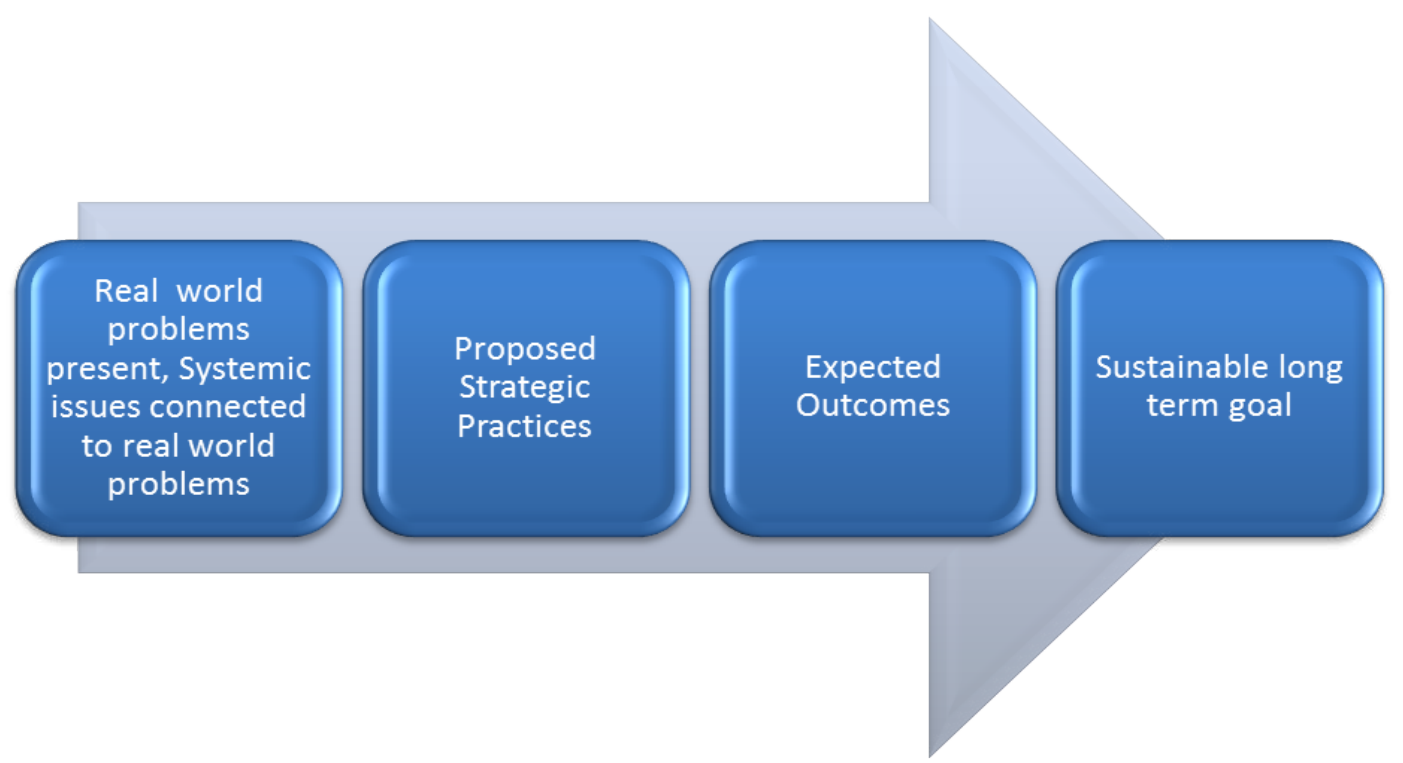

Figure 1: Four steps structure to evolve the strategic framework for real world problems and long term outcomes.

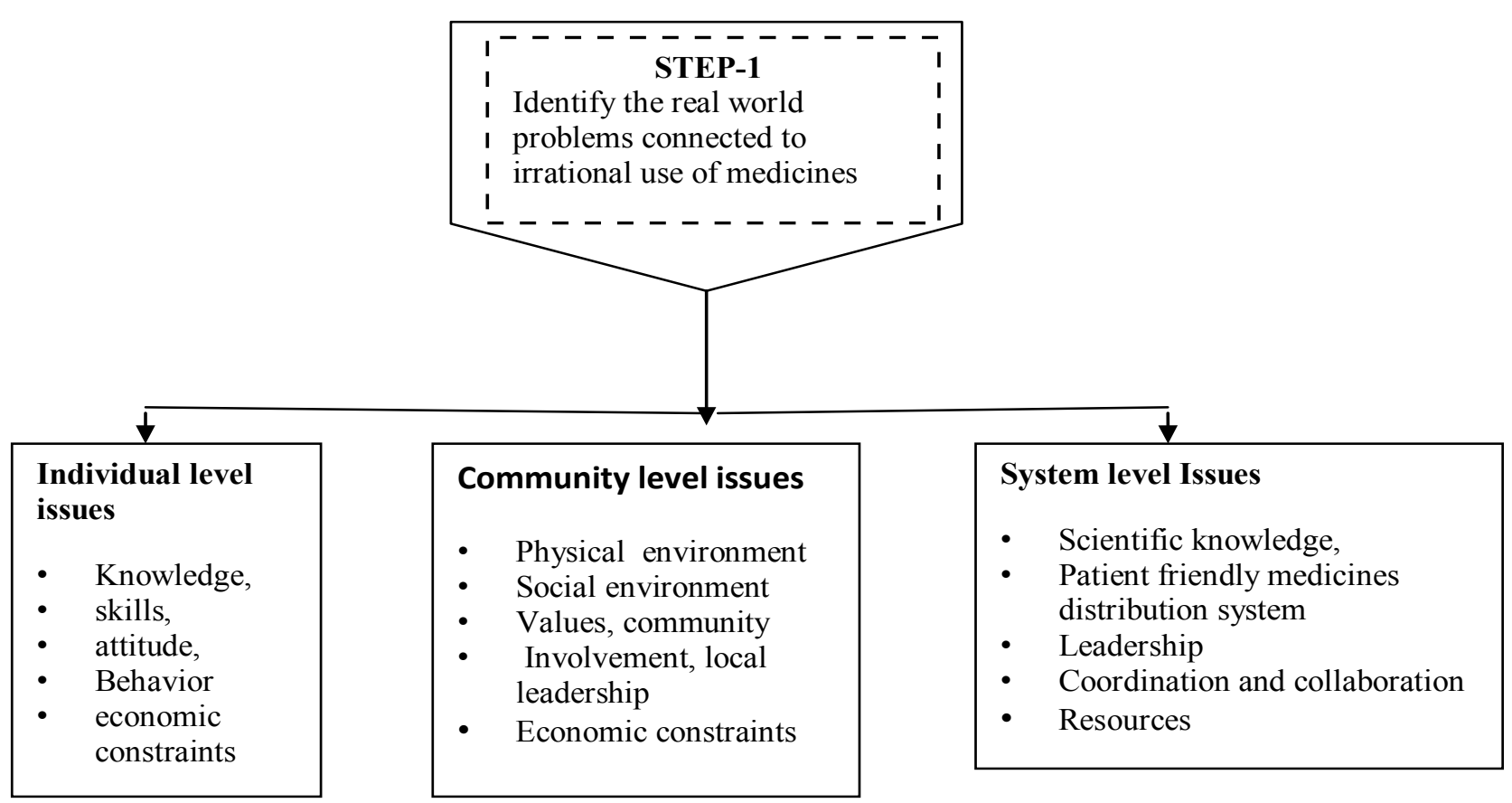

Figure 2: The strategic frame work flow chart to identify issues at different levels. 
Finally, theoretical logic framework model approach has been adopted to enhance the clarity, for developing the strategic framework. The Framework presents the rational basis for efforts related to 'promotion of rational use of medicines' and health problems by attempting together the following components such as: [11]

- Long-term problem(s) related to irrational usage of medicines.

- Factors that need to be addressed which contribute to the problem(s) related to irrational usage of medicines.

- Strategies and practices and supporting resources, which can be mobilized to address the factors and the problems of PRUM.

- Measurable outcomes and impacts that can be expected to result from implementing the strategies and practices towards PRUM and

- Long-term objectives and goals that can be achieved by effectively implementing strategies for promotion of rational use of medicines.

\section{Results}

The components of "Framework" are presented in four sections, organized sequentially into the components presented in the graphic depiction of its general structure shown in Figure 1 (Figure 1, Figure 2 and Figure 3).

\section{Step 1: Identification of attributing factors for PRUM}

To achieve the long term goal of promotion of rational use of medicines, following three levels of attributing factors have been identified i.e. individual level issues; community level issues and system level issues as follows.

Individual level issues: Individual level issues Encompass preventable morbidity and premature mortality experienced by irrational usage of medicines at individual's level. The factors at individual level could be knowledge, attitudes about the medicines usage pattern and practice of the same given range of economic and social constraints at individual level [13].

Community level issues: Community level issues may have range of community organizational and structural issues as mentioned in the flow diagram step-2, that may promote the rational usage of medicines in a sustainable fashion. The most important enabling factors are, local leadership and organizational structure for knowledge translation.

Systems level issues: Systems level issues encompass a wide variety of conceptual, organizational, structural and process-related variables. These variables include the availability of adequate resources to support the systems, the strategic practices aimed at the prob- lems and other contributing factors [14]. Our review indicates that a systems approach and systematic actions, need to apply broadly across all levels the purpose of implementing PRUM [15]. This finding applies specifically in using research and evaluation results to address gaps and weaknesses in implementation science and knowledge about the nature and extent of irrational usage of medicines. The three levels of issues are parallel and are interlinked with principal goals of PRUM for healthy life. Further, our review indicates that success in addressing PRUM will contribute to the achievement of the final outcome of the quality, healthy life.

\section{Step 2: Strategies to practices}

It is important to note that many of the strategies and practices may address several factors at the same time or in sequence, rather than only one factor. Based on our review, the following strategies have been proposed for strategic practices: Individual-level practices; community-level practices and systems-level strategic practices.

Individual-level strategic practices: It includes the knowledge and attitudes that individuals have about irrational usage of medicines and health risks. Approaches aiming to increase knowledge, promote positive attitudes, and improve skills that affect decisions about health-related behavior are successful at this level. A broad range of informational/educational methods and materials, dissemination channels and venues may be used such as written materials, including popular and professional publications, radio and television broadcasts, computer- and web-based technologies, mass media campaigns, and one-on-one or group-oriented education, counseling and training in schools, clinics, worksites and community settings. Strategies and practices may be aimed at a variety of individuals and groups of individuals, including, but not limited to medical practitioners, community leaders and pharmaceutical companies.

Effective efforts should reflect integrated approaches that address a combination of individual-level factors as well as their interactions with environmental factors that inhibit or support desired behaviours. In addition, health messages are more readily accepted if they do not conflict with existing cultural beliefs and practices, and take into account unique historical and cultural experiences of target audiences [16].

Community-level strategic practices encompass: $\mathrm{Com}$ munity-level strategic practices encompass physical environment (built), social and cultural characteristics of a community, economic, political and organizational conditions, specifically those that are not generally within the control at individual level. Our review indicates that the strategic practices at community level could reduce the irrational usage of medicines through promoting access to quality medicines. For example, a stricter le- 


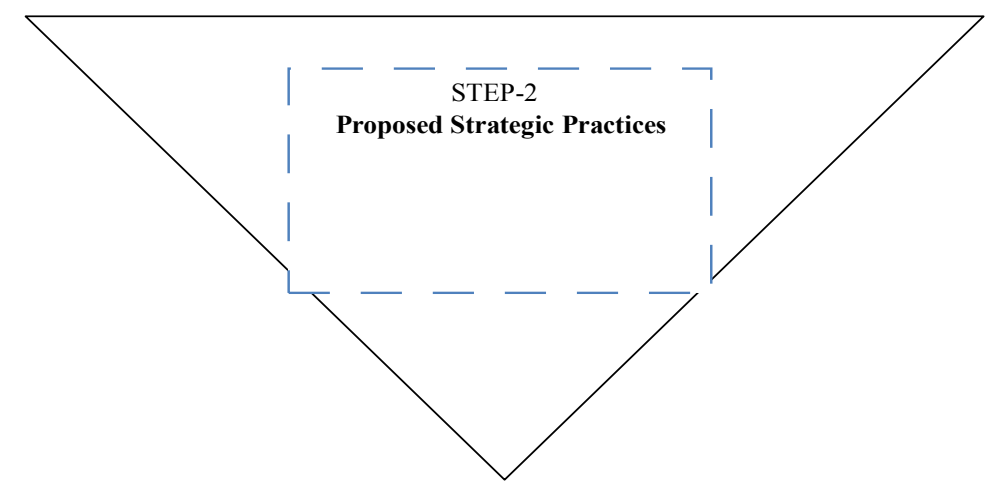

\begin{tabular}{|c|c|c|}
\hline $\begin{array}{l}\text { Individual level } \\
\text { - Strategies to promote healthy } \\
\text { practices and reduction of } \\
\text { irrational usage of medicines } \\
\text { - } \quad \text { Strategies to build skills } \\
\text { - Strategies to promote attitudes } \\
\text { conducive to healthy life with } \\
\text { minimal usage of medicines. } \\
\text { - Strategies to change the } \\
\text { knowledge /perception about } \\
\text { the usage of medications }\end{array}$ & 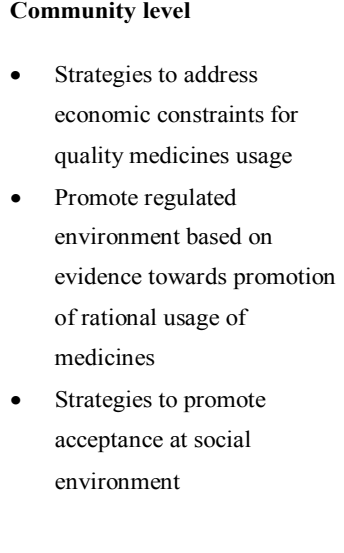 & 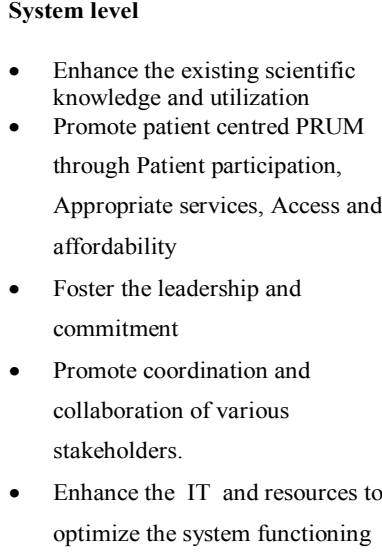 \\
\hline
\end{tabular}

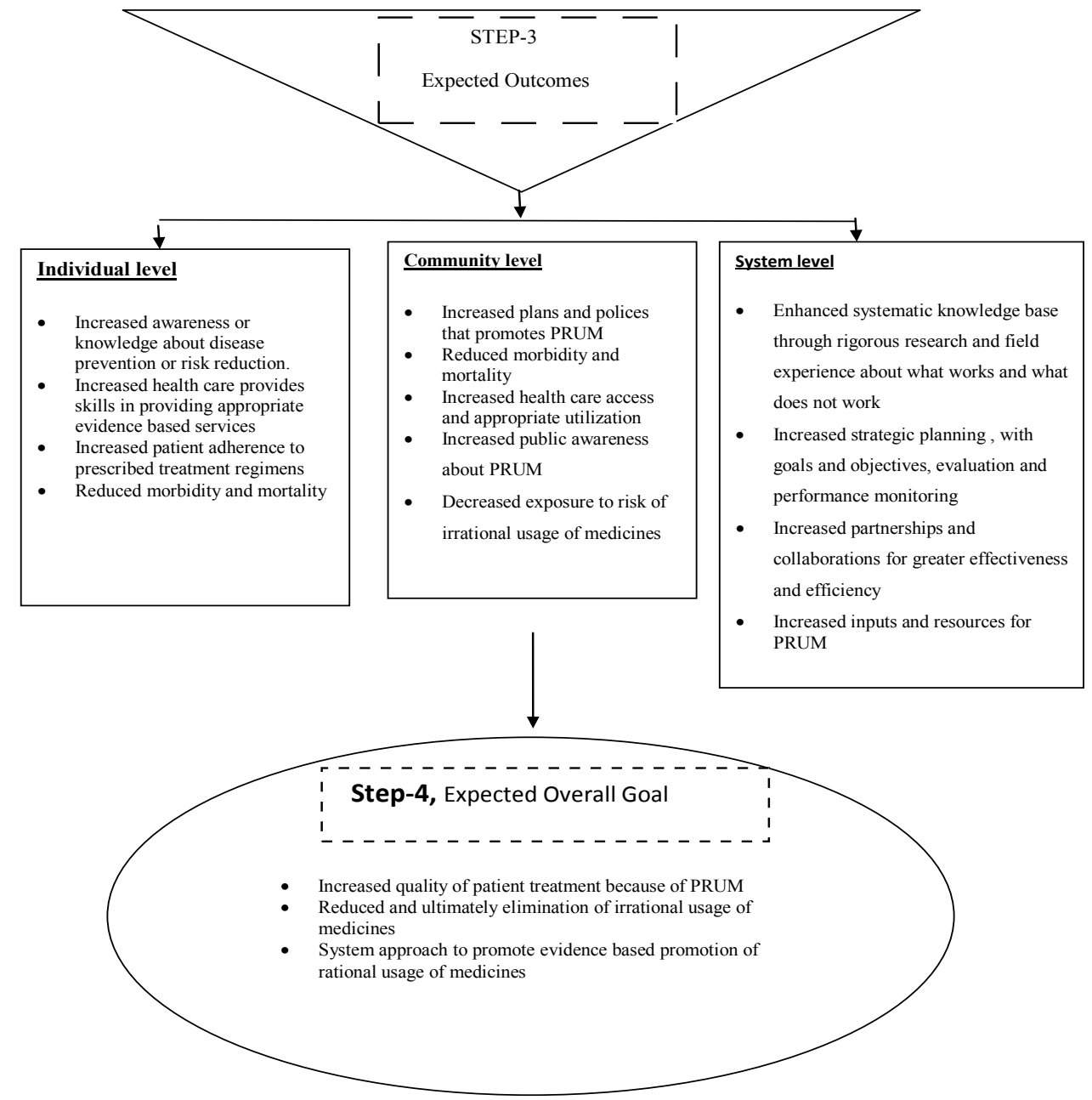

Figure 3: The strategic framework flow chart to identify issues at different levels. 
gal framework mandates the requirement of qualified medical practitioner prescriptions to disburse the medicines, promoting ethical practices among medical practitioners through involvement of professional bodies, enhancing regulatory mechanisms to check marketing of spurious medicines and advocacy regarding cultural values and norms that influence individual behaviour towards irrational consumption of medicines. To improve community level strategic practices, we propose the issues need to be further divided into specifics of physical environment, social environment including economic barriers, community values and ensuring enhanced community involvement.

Systems-level strategic practices: Systems-level strategic practices. We propose five components to ensure system level strategic practices.

1. First: Central and State governments should improve the infrastructure meant for efficient drug distribution system, should address increased and efficient staffing issues, allocation of enhanced and consistent funding sources, and mobilizing the resources especially [17].

Second: National, State and regional level coordination committees will have to be evolved to promote coordination, collaboration and strengthening existing system. This would allow pooling and leveraging of resources including expertise and talent to foster synergies that benefit all involved parties. These collaborations can be promoted by pharmaceutical companies in engagement with civil society organization and Governments.

Third: Provision of strong leadership and commitment. This needs to be exhibited by all stake holders through practice of evidence based public health programming. This includes building upon the best of existing system and utilizing emerging evidence of successful strategies and practices. Further, political will and leadership will have to be shown in implementing structured activities around expected outcomes at the State and central levels by employing performance assessment and evaluation results for continuous improvement. Improving ownership of the public health agencies will result in legislative and regulatory initiatives such as executive orders and other administrative mandates for promotion of rational usage of medicines.

Fourth: Promoting user-centered designs will enhance promotion of rational usage of medicines. Some of the methods include enhanced community-based participation and improving health care access and coverage.

Fifth: It is imperative to start and effectively implement a system to utilize knowledge about successful strategies and practices through increased dependency on research and evaluations [18]. A new system will have to be developed for collection, analysis and use of 'PRUM' data for performance monitoring and to improve quality of system. Further, research and development efforts that strengthen knowledge and understanding about the nature and extent of PRUM needs to be encouraged from Governments specifically to help weaker section populations [19].

Translating research into practice and policy and using the data from practice into research can address multi-faceted solutions towards PRUM.

\section{Step 3: Measuring outcomes and evaluating im- pacts}

We propose some of the measurable outcomes and impacts that might be expected to take place following implementation of the indicated strategies and practices (Table 1). We have categorized as outcomes impacts.

\section{Step 4: Long-term objectives and goals}

An important part of the strategic framework could be its focus on to encourage and support efforts that contribute to the long-term objectives and goals relevance to PRUM. The framework can be used to promote PRUM in different provinces, regions, communities and other stakeholders, who can use this framework to guide the selection of problems, factors and strategies/ practices that can be linked to short-term, intermediate- and long-term objectives and goals, based on identified outcomes and impacts. To the extent that strategies and practices result directly or indirectly in impacts on the determinants of health, and progress can be done toward the long-term goals of improving PRUM. Such efforts may necessitate a systems approach and a concerted effort to build and deploy evidence-based practice in order to promote continuous improvement based on coordinated and strategic application of the most current science and knowledge and to mobilize the resources and talents of all stakeholders involved.

\section{Discussion}

Several studies have pointed out the need for interventions at different levels which we propose to encompass at individual, community and system level. Some earlier studies have come up with experiences and models at hospitals and state levels $[21,22]$. Measures such as that of essential medicine lists have been advocated for and are serving as a point of guidance towards PRUM $[8,9,23]$. Also instituting pharmaco-vigilance measures with patient safety as the ultimate goal is aiding PRUM [24]. The proposed framework requires effective leadership and coordination within the broad health system spread across the nation. A realistic implementation of the proposed framework, requires critical mass of professionals, who can actively sustain engagement and efforts of all stakeholders [25]. The stakehold- 
Table 1: Outcomes and impacts of PRUM.

Individual-Level Outcomes and Impacts

- Increased awareness/knowledge about disease prevention, risk reduction and treatment and management for PRUM [20].

- Improved attitudes/beliefs conducive to health and health-seeking behaviors among communities

- Improved attitudes/beliefs among health care/human service providers and researchers conducive to meeting the needs of 'promotion of rational use of medicines'.

- Increased skills for promotion of rational use of medicines.

- Increased skills for public health/health care providers and other service professionals to provide culturally and linguistically appropriate services

- Increased patient satisfaction with patient-provider communications and interactions.

- Increased patient adherence to prescribed treatment regimens

- Increased engagement in/adoption of healthy lifestyle and appropriate health-seeking behaviors; reduced engagement in/adoption of risky behaviors

- Reduced morbidity and mortality

\section{Community-Level Outcomes and Impacts}

- Increased awareness/knowledge about irrational usage of medicines and health problems, among public health/health care providers and service professionals and in the general public.

- Increased health-conducive changes in community attitudes, values and norms on PRUM

- Increased number of active organizations and family or social networks that meet the social needs and promote the general health and well-being and importance of 'promotion of rational use of medicines' in the community (e.g., religious groups, social clubs, recreational and after-school programs)

- Increased health care access and appropriate utilization

- Increased number of plans and policies that promote and protect health and well-being at the community, state and national levels, in general by promoting 'promotion of rational use of medicines'.

- Reduced morbidity and mortality

\section{Systems-Level Outcomes and Impacts}

- Increased inputs, assets and other resources allocated for PRUM in general and for specific priorities

- Increased dedicated assets and other resources for PRUM.

- Increased formal partnerships and collaboration leading to coordination/leveraging of resources for greater efficiency, and enhanced effectiveness of PRUM.

- Increased strategic planning and implementation of plans, with clearly articulated goals and objectives, for PRUM.

- Increased integration of evaluation, performance measurement and monitoring, and continuous improvement in planning and implementation of 'promotion of rational use of medicines' efforts.

- Increased collection, dissemination and use of 'promotion of rational use of medicines' for planning, quality assurance and performance monitoring/improvement purposes (e.g., to assess whether clinical care guidelines for specific diseases are being employed consistently and appropriately, to address health care disparities)

- Increased knowledge development/science base about successful strategies and practices for improving PRUM.

- Increased dissemination and diffusion of evidence-based strategies and practice to improve PRUM and to reduce health problems.

- The identification of expected outcomes and impacts is an important part of the planning, implementation and evaluation processes needed in PRUM. Once desired or expected outcomes and impacts are identified, the process of determining performance measures or indicators of progress in achieving such outcomes and impacts can occur. With the identification and selection of performance measures or indicators of the expected outcomes or impacts, the effectiveness of the strategies and practices in producing the desired results can then be evaluated.

ers include individuals, communities, practitioners, health administrators, policy makers, academic and research institutions, state and central governments, faith- and community-based organizations, private industry, philanthropies and many others.

Earlier studies involving PRUM have not been clear in specifying desired or intended outcomes and impacts. In LMICs, evaluations have not been performed to determine if, any of such proposed strategies and practices can produce meaningful results [26]. The Framework proposed in this paper identifies and organizes a range of outcomes and impacts that might be expected, with reference to the contributing factors and the strategies and practices already discussed. Given the limitations and uncertainty of health environment, logic framework can guide even within a complex policy and decision-making environment. Nevertheless, this framework may be viewed as a dynamic, evolving one that provides direction for action rather than as a linear, predictable model for immediate problem solving and decision-making. In addition, the utility of this framework does not end 
with the achievement of some objectives and goals. Rather, results can and may be used to inform and to improve PRUM to tackle health problems [14]. Thus, any knowledge gained can be incorporated into the continuing efforts of all stakeholders. Such a process may help PRUM to consistently monitor and adjust program and policy efforts in ways that may result in greater effectiveness, efficiency and success [27]. The proposed Framework and its general structure can, thus, also serve as a direction for action in a number of ways, and for a variety of public and private entities.

\section{Limitations in Developing the Framework}

The four-step process outlined above may result in a strategic framework for addressing PRUM and health improvements and reducing, ultimately, eliminating the irrational usage of medicines and its health consequences. However, several points must be made regarding the task of identifying 'best' or evidence-based strategies and practices. First, many strategies and practices address multiple contributing factors and may contribute to multiple outcomes and impacts. There is not a one-to-one correspondence or a strictly linear relationship between contributing factors, strategies and practices and outcomes/impacts. Second, there is not adequate scientific evidence to demonstrate the effectiveness of all the strategies and practices that are considered in this paper. But more research may be done by other researchers to get more evidence and guide implementation science of health system.

\section{Conclusions}

This paper with proposed framework is intended to help promotion of rational use of medicines, focusing on range of stakeholders at different levels. The approach is a more systems-oriented and strategic, based on existing science and knowledge, to tackle the problems related to PRUM. In the long run, this strategic framework can help in multiple ways such as understanding perspectives involved in developing policies, can help deepen understanding about the multiple and complex relationships between several factors, and to identify areas and issues woven around the irrational usage of medicines. Most importantly, the proposed Framework is flexible and hence can evolve and improve, both in its structure and in its details as the experience accumulates. The best of the Framework can be in stimulating systematic planning, testing, and use of evidence-based strategies and practices that can really work. The proposed Framework is an attempt towards the road ahead for implementing evidence based public health practice in India. Efforts such as these constitute evidence based Public health practice and such efforts should guide planning and implementing health programs in developing countries such as India.

\section{Declaration}

The author declare no other conflict of interest.

\section{Funding}

The author declare no funding support for this review.

\section{References}

1. https://www.who.int/medicines/areas/rational_use/en/

2. World Health Organization (2012) The pursuit of responsible use of medicines: Sharing and learning from country experiences.

3. Dineshkumar B, Raghuram TC, Radhaiah G, Krishnaswamy K (1995) Profile of drug use in urban and rural India. Pharmacoeconomics 7: 332-346.

4. Mahajan R, Singh NR, Singh J, Dixit A, Jain A, et al. (2010) Current scenario of attitude and knowledge of physicians about rational prescription: A novel cross-sectional study. J Pharm Bioallied Sci 2: 132-136.

5. Kotwani A, Wattal C, Joshi PC, Holloway K (2012) Irrational use of antibiotics and role of the pharmacist: An insight from a qualitative study in New Delhi, India. J Clin Pharm Ther 37: $308-312$

6. Ganguly NK, Arora NK, Chandy SJ, Fairoze MN, Gill JP, et al. (2011) Rationalizing antibiotic use to limit antibiotic resistance in India. Indian J Med Res 134: 281-294.

7. Laing R, Hogerzeil H, Ross-Degnan D (2001) Ten recommendations to improve use of medicines in developing countries. Health Policy Plan 16: 13-20.

8. Ratanawijitrasin S, Soumerai SB, Weerasuriya K (2001) Do national medicinal drug policies and essential drug programs improve drug use?: A review of experiences in developing countries. Soc Sci Med 53: 831-844.

9. Laing R, Waning B, Gray A, Ford N, 't Hoen E (2003) 25 years of the WHO essential medicines lists: Progress and challenges. Lancet 361: 1723-1729.

10. Haggerty JL, Yavich N, Bascolo EP (2009) A framework for evaluating primary health care in Latin America. Rev Panam Salud Publica 26: 377-384.

11. Claxton K, Sculpher M, Drummond M (2002) A rational framework for decision making by the National Institute for Clinical Excellence. Lancet 360: 711-715.

12. Baxter S, Killoran A, Kelly MP, Goyder E (2010) Synthesizing diverse evidence: The use of primary qualitative data analysis methods and logic models in public health reviews. Public Health 124: 99-106.

13. Jackson SH, Mangoni AA, Batty GM (2004) Optimization of drug prescribing. Br J Clin Pharmacol 57: 231-236.

14. Kriska M, Halko J, Turcani P (1999) General principles of drug evaluation within the framework of drug policy. Bratisl Lek Listy 100: 490-493.

15. Ritter A, Bammer G (2010) Models of policy-making and their relevance for drug research. Drug Alcohol Rev 29: 352-357.

16. Kreuter MW, McClure SM (2004) The role of culture in health communication. Annu Rev Public Health 25: 439-455.

17. Trouiller $\mathrm{P}$, Torreele E, Olliaro $\mathrm{P}$, White $\mathrm{N}$, Foster $\mathrm{S}$, et al. (2001) Drugs for neglected diseases: A failure of the market and a public health failure? Trop Med Int Health 6: 945-951. 
18. Guillemot D, Maison P, Carbon C, Bouvenot G, Imbs JL (1999) Development of pharmaco-epidemiology in France. Presse Med 28: 493-499.

19. De Vries TP (1993) Presenting clinical pharmacology and therapeutics: A problem based approach for choosing and prescribing drugs. Br J Clin Pharmacol 35: 581-586.

20. de Menil V, Cohen Am (2009) Rational use and rationale for use: Psychiatric medication at an Argentine institution for intellectual disability. Transcult Psychiatry 46: 651-671.

21. Chaudhury RR, Parameswar R, Gupta U, Sharma S, Tekur $U$, et al. (2005) Quality medicines for the poor: Experience of the Delhi programme on rational use of drugs. Health Policy Plan 20: 124-136.

22. Singh I, Mittal R, Shafiq N, Bharati B, Nigah RK, et al. (2010) A drug utilization study to provide background data for bringing amendments in the drug dispensing policy of a pediatric referral center. Pharmacoepidemiol Drug Saf 19: 393-399.
23. Kar SS, Pradhan HS, Mohanta GP (2010) Concept of essential medicines and rational use in public health. Indian $\mathrm{J}$ Community Med 35: 10-13.

24. Olsson S, Pal SN, Stergachis A, Couper M (2010) Pharmacovigilance activities in 55 low- and middle-income countries: A questionnaire-based analysis. Drug Saf 33: 689703.

25. Nichter M, Vuckovic N (1994) Agenda for an anthropology of pharmaceutical practice. Soc Sci Med 39: 1509-1525.

26. Lopert R, Rosenbaum S (2007) What is fair? Choice, fairness, and transparency in access to prescription medicines in the United States and Australia. J Law Med Ethics 35: 643-656.

27. Fijn R, Lenderink AW, Egberts AC, Brouwers JR, De JongVan DenBerg LT (2001) Assessment of indicators for hospital drug formulary non-adherence. Eur J Clin Pharmacol 57: 677-684. 